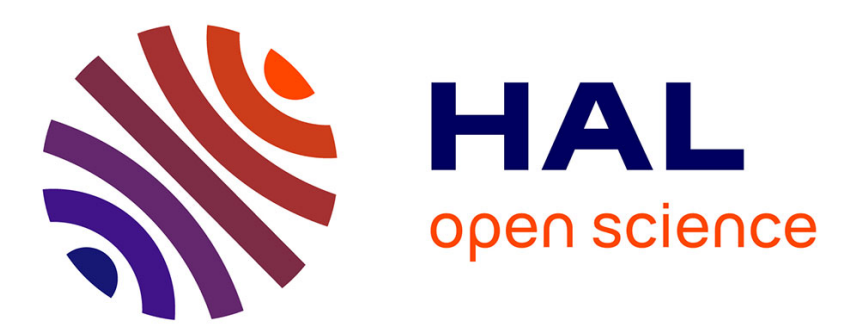

\title{
Limited-Bandwidth Fractional Differentiator: Synthesis and Application in Vibration Isolation
}

Pascal Serrier, Xavier Moreau, Alain Oustaloup

\section{To cite this version:}

Pascal Serrier, Xavier Moreau, Alain Oustaloup. Limited-Bandwidth Fractional Differentiator: Synthesis and Application in Vibration Isolation. Advances in Fractional Calculus: Theoretical Developments and Applications in Physics and Engineering, 2007, pp.287-302. hal-00326406

\section{HAL Id: hal-00326406 https://hal.science/hal-00326406}

Submitted on 2 Oct 2008

HAL is a multi-disciplinary open access archive for the deposit and dissemination of scientific research documents, whether they are published or not. The documents may come from teaching and research institutions in France or abroad, or from public or private research centers.
L'archive ouverte pluridisciplinaire HAL, est destinée au dépôt et à la diffusion de documents scientifiques de niveau recherche, publiés ou non, émanant des établissements d'enseignement et de recherche français ou étrangers, des laboratoires publics ou privés. 


\title{
LIMITED-BANDWIDTH FRACTIONAL DIFFERENTIATOR: SYNTHESIS AND APPLICATION IN VIBRATION ISOLATION
}

\author{
Pascal Serrier, Xavier Moreau and Alain Oustaloup
}

LAPS-UMR 5131 CNRS, Université Bordeaux1 - ENSEIRB, 351 cours de la Libération. 33405 TALENCE cedex, France,

\{pascal.serrier, xavier.moreau, alain.oustaloup\}@laps.u-bordeaux1.fr

\begin{abstract}
The use of fractional differentiation in vehicle suspension design has many interests. This paper presents a hydropneumatic suspensions design method based on fractional differentiation. Once an hydraulic structure has been chosen for the suspension, it is possible to calculate the values of all technological parameters, so that the suspension force - deflection transfer function is a band-limited fractional differentiator. The combination of the CRONE control methodology and of hydropneumatic technology leads to remarkable performances of robustness.
\end{abstract}

\section{Key words}

Fractional differentiation, Hydropneumatic technology, CRONE control, Hydropneumatic test bench

\section{Introduction}

For a long time, fractional derivative was not used because of the lack of physical signification of this concept and the lack of means to synthesize and achieve fractional differentiators. Nowadays, fractional derivative is used in numerous applications, such as heat transfer phenomena [1], dielectric polarization [2] and vibration isolation [3].

This paper presents, in vibration isolation context, two structures of hydropneumatic components allowing to achieve a suspension whose Forcedeflection transfer function is a given limited-bandwidth fractional 
differentiator. In a more general context, two means to achieve limitedbandwidth fractional differentiator in hydropneumatic technology are proposed.

The first part reminds the formulation of vibration isolation problem as robust control synthesis problems and the interest of fractional derivative in vibration isolation.

Through the example of a hydraulic test bench, the second part presents the synthesis of a limited-bandwidth fractional differentiator to satisfy given specifications in a vibration isolation context. Two structures are proposed to achieve a suspension in hydropneumatic technology. A method to determine the corresponding technological parameters is described in each case.

The third part shows the simulated performances obtain with the test bench, taking into account the influence of sprung mass variation on the hydropneumatic components.

The last part concerns the future development of this work.

\section{Fractional derivative in vibration isolation}

\subsection{From vibration isolation to robust control synthesis}

Vibration isolation is a usual mechanical problem which consists in limiting vibration transmission between a source and one or some systems. A solution is to isolate the system from the source by using a vibration isolator, also called suspension. This problem can be formalized as a usual problem of control synthesis in the field of Automatics.

Some previous works [4] have shown, from a one-degree-of-freedom model (figure 1), that a suspension which develops a force $u(t)$ that is links to its deflection $z_{10}(t)$ by the relation:

$$
U(s)=-D(s) Z_{10}(s),
$$

naturally makes a feedback control around the static equilibrium position.

The block diagram (figure 2) which is issued from the modeling clearly shows that the suspension has the same role that the controller of a control loop, that the displacement and force solicitations $\left(z_{0}(t), f_{0}(t)\right)$ can be considered as input and output perturbations which act on the plant. The plant is a double integrator whose transitional pulsation depends on the sprung mass.

The open loop transfer function $\beta(s)$ is:

$$
\beta(s)=D(s) G(s) \text {, with } G(s)=\frac{1}{M s^{2}} .
$$




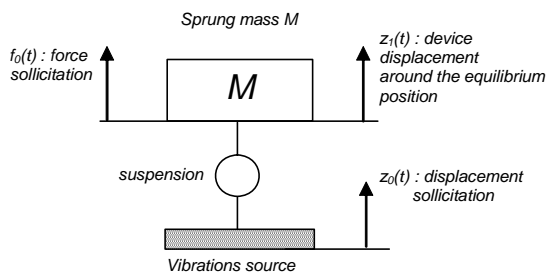

Fig. 1. One degree of freedom (DOF) model

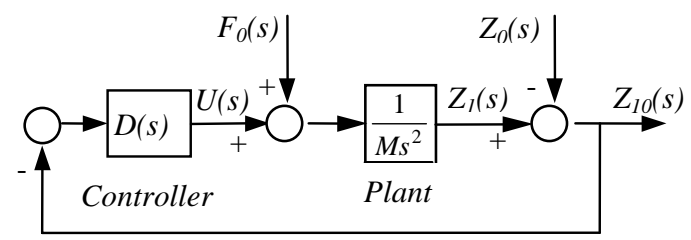

Fig. 2. One degree of freedom model block diagram

Thus, suspension design can be made by using the classic control synthesis method. In particular, when parameters uncertainties (especially mass variation) are considered, the problem becomes a robust control synthesis problem.

\subsection{The CRONE suspension}

The CRONE suspension is a suspension whose synthesis is based upon CRONE control one [5]. The CRONE control allows to obtain the stability degree robustness in spite of parameters uncertainties. The $2^{\text {nd }}$ generation CRONE control is used when either parameter uncertainties correspond only to plant gain variations or when parameter and controller uncertainties compensate for themselves and lead to a behavior like the one that will be develop below. In both cases, the open loop phase remains the same around the gain crossover pulsation $\omega_{u}$.

In the particular case of this article, the suspension force-deflection transfer function is a limited bandwidth fractional differentiator [6], namely:

$$
D(s)=D_{0}\left(\frac{1+\frac{s}{\omega_{b}}}{1+\frac{s}{\omega_{h}}}\right)^{m},
$$


where $D_{0}$ is the static gain, $\omega_{b}$ and $\omega_{h}$ are the low and high transitional pulsations and where $m$ is the fractional order, that means that $m$ may be not non-integer.

$D_{0}, m, \omega_{b}$ and $\omega_{h}$ are in the CRONE method the high level synthesis parameters. These four parameters are determined from the specification sheets.

Bode diagrams of this suspension are given figure 3 . The phase is constant between the pulsations $\omega_{A}$ and $\omega_{B}$.

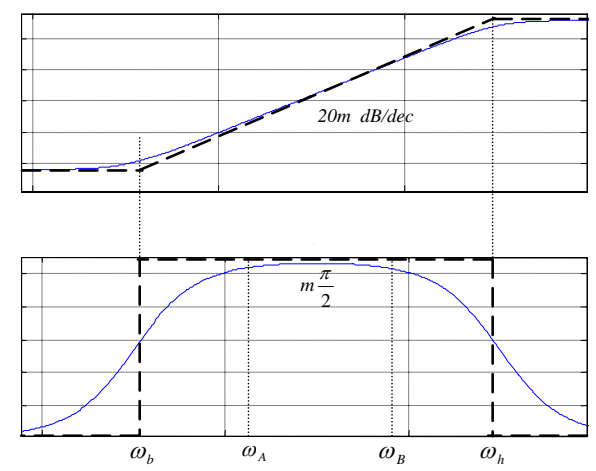

Fig. 3. Bode diagrams of a limited-bandwidth fractional differentiator

To achieve a real hydropneumatic device whose transfer function is a limited bandwidth fractional differentiator, or only to simulate a fractional differentiator, it is necessary to synthesize a rational approximation of the fractional differentiator. A method to do this is proposed in [6].

The desired transfer function $D(s)$ can be approximated by $N$ poles and $N$ zeros. This approximation $D_{N}(s)$ is given by [6]:

$$
D_{N}(s)=D_{0} \prod_{i=1}^{N}\left(\frac{1+\frac{s}{\omega_{i}^{\prime}}}{1+\frac{s}{\omega_{i}}}\right),
$$

where $\omega_{i}^{\prime}$ and $\omega_{i}$ are recursively distributed through the recursive coefficients $\alpha$ and $\eta$ which are defined by [6]:

$$
\frac{\omega_{i+1}^{\prime}}{\omega_{i}^{\prime}}=\frac{\omega_{i+1}}{\omega_{i}}=\alpha \eta>1, \frac{\omega_{i}}{\omega_{i}^{\prime}}=\alpha>1 \text { and } \frac{\omega_{i+1}^{\prime}}{\omega_{i}}=\eta>1 \text {. }
$$

In hydropneumatic technology, the CRONE suspension is made of hydraulic accumulator (capacitive components, C, which cont ain oil and gas, nitrogen, separated by an impermeable diaphragm) and hydraulic dampers (dissipative components, R). 
Two structures are considered in the following part: a parallel arrangement of $\mathrm{R}$ and $\mathrm{C}$ components in series (RC cells) (figure 4), and gamma arrangement (figure 5).

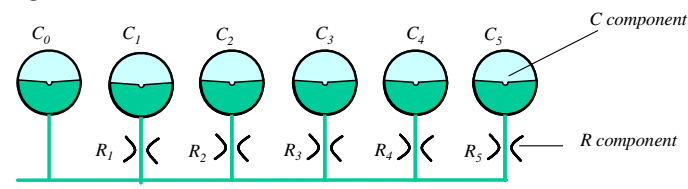

Fig. 4. Parallel arrangement of RC cells

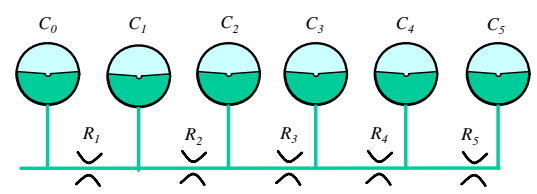

Fig 5. Gamma arrangement

\section{CRONE hydropneumatic test bench}

This part uses a CRONE hydropneumatic test bench as an example to analyse the previous structures.

\subsection{Description}

The CRONE test bench allow to study the free evolution of a mass $(M)$ after a release test. The mass is mechanically link to a hydraulic simple effect jack (figure 6). The minimal mass of $75 \mathrm{~kg}$ can be increased by additional masses. So, $M$ can vary between $75 \mathrm{~kg}$ and $150 \mathrm{~kg}$.

The suspension jack is connected to a two parts hydraulic circuit (figure 6). The first part is composed of a pump equipped with a make and brake circuit and a proportional valve. Its aim is to maintain the mass $M$ at a fixed height independently of the mass value thanks to a control feedback. The second is composed of two change over valves which allow to select either a parallel arrangement of two cells including one RC cell $(N=1, N$ is the number of RC cells), or a parallel arrangement of six cells including five RC cell $(N=5)$ or a gamma arrangement of six cells. 


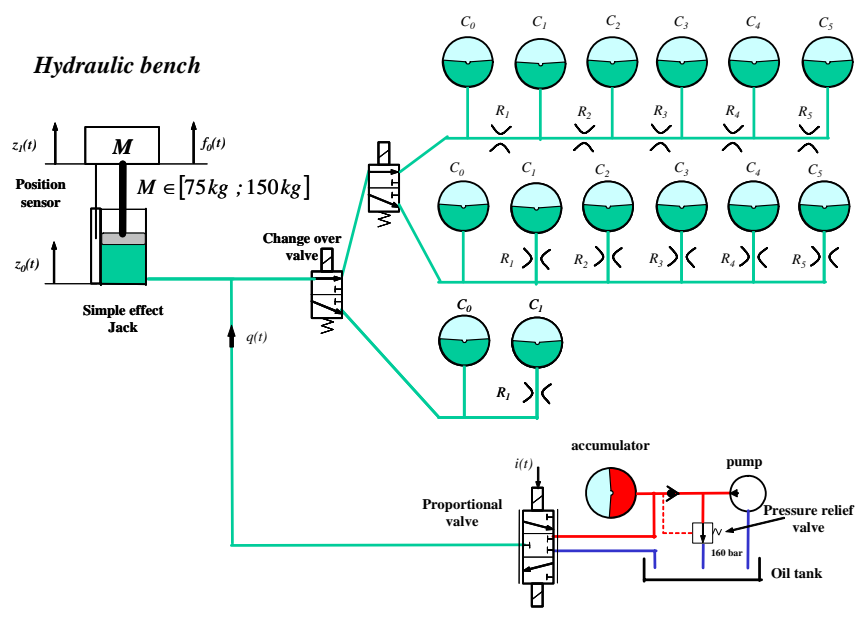

Fig. 6. Hydraulic diagram of the CRONE test bench

The associated control diagram is presented figure 7. The external loop which regulates the static equilibrium position at a value equal to half stroke of the jack has the same rapidity as the self-leveller device of a hydropneumatic suspension. This rapidity is characterized by an open-loop gain cross-over frequency of $0.1 \mathrm{rad} / \mathrm{s}$. The internal loop has a rapidity characterised by an open-loop gain cross-over frequency of $6 \mathrm{rad} / \mathrm{s}$, the same rapidity as the vertical mode of a usual vehicle. So, both loops are dynamically uncoupled. That is why only the internal loop is considered in the following parts of this article.

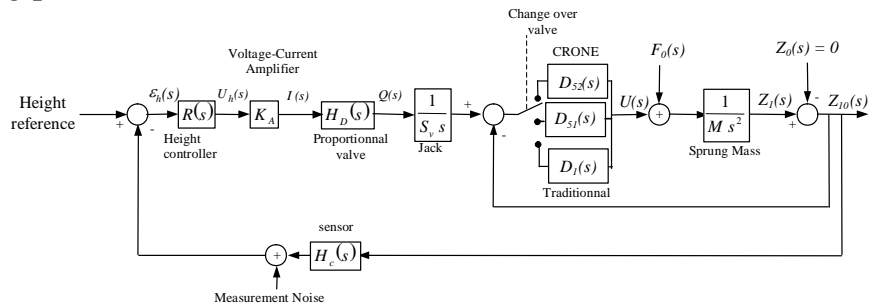

Fig. 7. Control diagram of the CRONE test bench

\subsection{CRONE suspension achievement in hydropneumatic technology}

The following steps are necessary to determine the technological parameters of the hydropneumatic components: 
From the specification sheets (rapidity, stability...), the suspension desired transfer function $D_{N}(s)$ is determined, according to the CRONE control methodology [5]. This ideal transfer function is characterized by the four high level synthesis parameters.

A rational approximation with $N$ poles and $N$ zeros is then established thanks to relation (5).

Two sets of relations (one for each structures: parallel arrangement of RC cells or gamma arrangement), which are established in the two following paragraphs, link the $N$ poles and $N$ zeros to the $2 N+1$ physical parameters ( $N$ resistances and $N+1$ capacities) of the hydropneumatic suspension.

Two others relations (which are detailed in the next part) allow to obtain the $2(2 N+1)$ technological parameters from the physical parameters.

Finally, if the suspension is achieve with five RC cells $(N=5)$, twenty two technological parameters are obtained from the four high level synthesis parameters.

\subsection{Relations between technological parameters and the poles and the zeros recursive distribution}

\section{Parallel Arrangement of RC Cells}

The input hydraulic impedance of each parallel arrangement is characterized by an expression of the form:

$$
\frac{P_{e}(s)}{Q_{e}(s)}=\frac{1}{C_{0} s+\sum_{i=1}^{N} \frac{1}{R_{i}+\frac{1}{C_{i} s}}},
$$

where $P_{e}(s)$ and $Q_{e}(s)$ are the pressure and the flow at the input point, where $R_{i}$ and $C_{i}$ are the $\mathrm{i}^{\text {th }}$ cell resistance and capacity whose expression is obtained by linearizing the hydraulic accumulator pressure-volume characteristic around the equilibrium point. The equilibrium point is defined by the static pressure $P_{S}$ and the gas volume $V_{S i}$. The capacity is thus given by [3]:

$$
\frac{1}{C_{i}}=-\left.\frac{\partial P}{\partial V}\right|_{\substack{P=P s \\ V=V s i}}=\gamma \frac{P_{s}}{V_{s i}},
$$

$\gamma$ is the thermodynamic coefficient which characterizes the gas evolution ( $\gamma=1$ for an isotherm evolution, $\gamma=1.4$ for an adiabatic evolution). Knowing that the product between the pressure and gas volume is constant ( $P V=c s t)$, the gas volume $V_{S i}$ can be expressed with the $\mathrm{i}^{\text {th }}$ accumulator calibration pressure $P_{0 i}$ and the volume $V_{0 i}$ (gas initial volume), namely: 


$$
V_{s i}=\frac{P_{0 i}}{P_{s}} V_{0 i},
$$

so the capacity $C_{i}$ expression:

$$
C_{i}=\frac{P_{0 i} V_{0 i}}{\gamma P_{s}^{2}} .
$$

The hydraulic resistor $R_{i}$ is dimensioned so that the flow is laminar [7]:

$$
R_{i}=\frac{128}{\pi} \mu \frac{l_{R i}}{d_{R i}^{4}},
$$

where $\mu$ is the oil dynamic viscosity, $l_{R i}$ and $d_{R i}$ are respectively the hydraulic resistor length and diameter (figure 8 ).

Expressions (9) and (10) define the relations between physical hydropneumatic parameters and technological parameters.

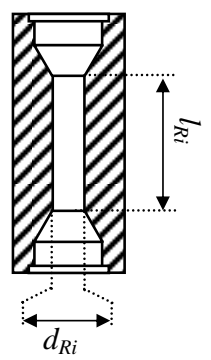

Fig. 8. Hydraulic resistance

More over, if the pressure drop due to the valve is considered negligible, the arrangement input pressure $P_{e}(s)$ is equal to the jack pressure. That is why the pressure $P_{e}(s)$ is linked to the force $U(s)$, which is applied on the mass $M$ by the jack, by the relation:

$$
P_{e}(s)=\frac{U(s)}{S_{v}},
$$

where $S_{V}$ is the jack section.

When the self-leveller device is not in action, the arrangement input flow $Q_{e}(s)$ depends on the jack displacement $Z_{10}(s)$ :

$$
Q_{e}(s)=S_{v} s Z_{10}(s) \text {. }
$$

The introduction of relation (11) and (12) in equation (6) leads to the forcedeflection transfer function expression $D_{N}(s)$, namely: 


$$
D_{N}(s)=-\frac{U(s)}{Z_{10}(s)}=S_{v}^{2} s \frac{P_{e}(s)}{Q_{e}(s)}=\frac{S_{v}^{2} s}{C_{0} s+\sum_{i=1}^{N} \frac{1}{R_{i}+\frac{1}{C_{i} s}}} .
$$

By dividing the denominator by $S_{v}^{2} s$, the expression (13) becomes:

$$
D_{N}(s)=\frac{1}{\frac{C_{0}}{S_{v}^{2}}+\sum_{i=1}^{N} \frac{1}{R_{i} S_{v}^{2} s+\frac{S_{v}^{2}}{C_{i}}}},
$$

expression of the form:

$$
D_{N}(s)=\frac{1}{\frac{1}{k_{0}}+\sum_{i=1}^{N} \frac{1}{b_{i} s+k_{i}}},
$$

by introducing:

$$
k_{0}=\frac{S_{v}^{2}}{C_{0}}, k_{i}=\frac{S_{v}^{2}}{C_{i}} \text { and } b_{i}=S_{v}^{2} R_{i} \text {, }
$$

where $k_{0}$ and $k_{i}$ are homogeneous to stiffnesses, which are expressed in $\mathrm{N} / \mathrm{m}$, and where $b_{i}$ are homogeneous to viscous damping coefficients which are expressed in $\mathrm{Ns} / \mathrm{m}$. The hydraulic arrangement equivalent mechanical diagram is given figure 9 .

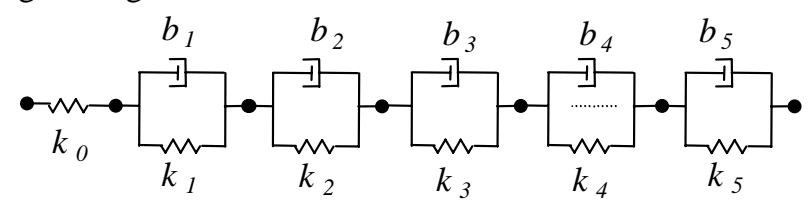

Fig. 9. Hydraulic arrangement equivalent mechanical diagram

Finally, $D_{N}(s)$ can be written:

$$
D_{N}(s)=\frac{1}{\frac{1}{k_{0}}+\sum_{i=1}^{N} \frac{1 / b_{i}}{s+\omega_{z i}}},
$$

by introducing

$$
\omega_{z i}=\frac{k_{i}}{b_{i}} .
$$

In order to establish the relations between the mechanical parameters $k_{i}$ and $b_{i}$ (or hydropneumatic $C_{i}$ and $R_{i}$ ) and the recursive distribution of the transitional frequencies $\omega_{i}$ and $\omega_{i}^{\prime}$, the inverse of relation (17), namely: 


$$
D_{N}^{-1}(s)=\frac{1}{k_{0}}+\sum_{i=1}^{N} \frac{1 / b_{i}}{s+\omega_{z i}},
$$

is interpreted as the decomposition in simple elements of the inverse of relation (4), that is to say:

$$
D_{N}^{-1}(s)=\frac{1}{D_{0}} \prod_{i=1}^{N}\left(\frac{1+\frac{s}{\omega_{i}}}{1+\frac{s}{\omega_{i}^{\prime}}}\right)=\frac{1}{D_{0}} \prod_{i=1}^{N} \frac{\omega_{i}^{\prime}}{\omega_{i}}+\sum_{i=1}^{N} \frac{A_{i}}{s+\omega_{i}^{\prime}},
$$

with

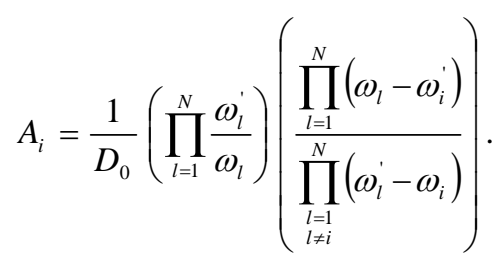

Member with member identification of the relations (19) and (20) makes it possible to determine the mechanical parameters $k_{0}, b_{i}$ and $k_{i}$, namely:

$$
k_{0}=D_{0} \prod_{i=1}^{N} \frac{\omega_{i}}{\omega_{i}^{\prime}}, \quad b_{i}=\frac{1}{A_{i}} \text { and } k_{i}=\omega_{i}^{\prime} b_{i},
$$

as well as hydropneumatic parameters $C_{0}, R_{i}$ and $C_{i}$, that is to say, taking into account the relations (16).

Lastly, the technological parameters such as the calibration pressure $P_{0 i}$ and the volume $V_{0 i}$ of each accumulator, the diameter $d_{R i}$ and the length $l_{R i}$ of each resistance, are deduced from relations (9) and (10) by taking into account the technological constraints associated to each component.

\section{Gamma Arrangement}

For simplification purpose, the following relations are established in the case of 6 hydro pneumatic accumulators and 5 hydraulic resistors. It is, of course, possible to extend these relations to more or less components. by:

The input hydraulic impedance of the gamma arrangement is characterized 


$$
\frac{P_{e}(s)}{Q_{e}(s)}=\frac{1}{C_{0} s+\frac{1}{R_{1}+\frac{1}{C_{1} s+\frac{1}{\ldots+\frac{1}{\ldots s+\frac{1}{R_{5}+\frac{1}{C_{5} s}}}}}}} .
$$

To compare this expression to the desired hydraulic impedance, which is obtained by dividing relation (4) by $S_{v}{ }^{2} s$, the two transfer functions have to be written under the same form. The most valuable form for comparison is continuous fraction.

Expressions (4) and (25) can be converted to the same simple continuous fraction form, namely:

$$
\frac{P_{e}(s)}{Q_{e}(s)}=\frac{1}{A_{0} s+B_{0}+\frac{1}{A_{2} s+B_{2}+\frac{1}{A_{3} s+B_{3}+\frac{1}{A_{4} s+B_{4}+\frac{1}{A_{5} s+B_{5}}}}}} .
$$

Member to member identification makes it possible to determine the mechanical parameters $k_{0}, b_{i}$ and $k_{i}$ in the case of gamma arrangement as well as hydropneumatic parameters $C_{0}, R_{i}$ and $C_{i}$, that is to say, taking into account the relations (16).

Technological parameters are obtained thanks to relations (9) and (10).

\subsection{Important note}

Capacities $C_{i}$ depend on the static pressure (9). The static pressure $P_{S}$ can be expressed according to the weight $M g$ and of the jack section $S_{V}$, namely:

$$
P_{s}=\frac{M g}{S_{v}} .
$$

By replacing in relation (9) $P_{S}$ by its expression (24), one notes that capacities $C_{i}$, and thus stiffnesses $k_{i}$, depend on the square of the sprung mass $M$, that is to say:

$$
C_{i}=\frac{S_{v}^{2} P_{0 i} V_{0 i}}{\gamma(M g)^{2}} \text { and } k_{i}=\gamma \frac{(M g)^{2}}{P_{0 i} V_{0 i}} \text {. }
$$


Thus, the variations or uncertainties of the sprung mass $M$ not only affect the plant $G(s)$ as defined in paragraph 2, but also the real form $D_{N}(s)$ of the controller because of the relations between the physical parameters and the parameters of $D_{N}(s)$.

This result leads to a new problematic in automatic control. Usually, controller uncertainties are not taken into account because they are much smaller than the plant uncertainties. In the particular case of an hydropneumatic achievement, controller uncertainties are not negligible any more.

A method to take into account the characteristics related to hydropneumatic technology during the synthesis of the limited-bandwidth fractional differentiator has been developed in [8]. It is based on two remarkable properties of the limited bandwidth fractional differentiator achieved in hydropneumatic technology. Indeed, it was shown that, in this case, the recursive parameters $\alpha$ and $\eta$ are independent of the variations of the mass $M$. So, the fractional order asymptotic behaviour of $D_{N}(s)$, characterized for the Gain diagram by a slope of $\mathrm{m} 20 \mathrm{~dB} / \mathrm{dec}$ and for the phase diagram by a phase blocking of $m \frac{\pi}{2} \mathrm{rad}$, is not modified; only the frequency domain where this asymptotic behaviour exists is relocated towards the high frequencies when the mass increases (and reciprocally towards the low frequencies when it decreases). Moreover, it is shown that the open-loop crossover frequency can remain insensitive to the variations of $M$. It is then possible to take into account these two results in the determination of the high level synthesis parameters of the limited bandwidth fractional differentiator to translate them, not only by the robustness of the degree of stability (intrinsic property with CRONE approach), but also by the robustness of the rapidity (intrinsic property to hydropneumatic technology).

From the following specifications [7]:

- for the rapidity, an open-loop cross-over frequency $\omega_{u}$ of $6 \mathrm{rad} / \mathrm{s}$;

- for the stability, a phase margin $M_{\Phi}$ of $45^{\circ}$;

- for the uncertainties, $M \in[75 \mathrm{~kg} ; 150 \mathrm{~kg}]$,

the four high level synthesis parameters of the ideal form $D(s)$ for the minimal mass $M=75 \mathrm{~kg}$ are calculated [7], namely:

and

$$
m=0.5, \omega_{b}=0.1 \mathrm{rad} / \mathrm{s}, \omega_{h}=90 \mathrm{rad} / \mathrm{s}
$$

$$
D_{0}=349 \mathrm{~N} / \mathrm{m}
$$

Then, thanks to the relations between the four high level synthesis parameters and the $2 \mathrm{~N}$ real form parameters, the transitional frequency and 
the recursive distribution can be calculated, always considering the minimal mass, with $N=5$.

In the case of a parallel arrangement of RC cells, the physical parameters are calculated from relations (22) and in the case of a gamma arrangement from relations (23) and (24).

Lastly, from the jack section, and the relations (9) and (10), the technological parameters are deduced. (Volumes $V_{0 i}$ come from constructor data sheets).

The numeric values of all parameters can be found in [8].

\subsection{Performances}

Within the framework of a comparative study, the parameters of arrangement with two cells (whose RC, $\mathrm{N}$ is $=1$ for the traditional suspension) are calculated starting from the same specifications as previously. Thus, for the minimal mass of $75 \mathrm{~kg}$, the three systems present the same dynamic.

Figure 9 presents the frequency responses obtained with the traditional suspension ((a), (c) and (e)) and CRONE suspension ((b), (d) and (f)) for the two extreme values of the sprung mass $M$ (blue dotted line: $M=75 \mathrm{~kg}$, in green $M=150 \mathrm{~kg}$ ). The results obtain with the parallel arrangement of RC cells and with the gamma arrangement are exactly the same. This first significant result explains why only one diagram is shown the CRONE suspension and not one for each structure.

The transfer functions $D_{1}(s)$ (traditional suspension (a)) and $D_{5}(s)$ (CRONE suspension (b)) Bode diagrams highlights the influence of a mass $M$ increase. Indeed, in both cases, the static gain $D_{0}$ increases and the transitional frequencies is relocated towards the high frequencies without the maximum in lead-phase not being modified. Thus, the length of the frequency domain which characterizes the second generation CRONE control [5] is dimensioned so that the open-loop cross-over frequency belongs to this fractional order asymptotic behavior whatever the mass $M$ values is between 75 and $150 \mathrm{~kg}$. This result is illustrated by the open-loop Bode diagrams (figure 9.d) and Black-Nichols loci (figure 9.f) in the case of CRONE suspension.

Moreover, around the cross-over frequency $\omega_{u}$ (figure 9.d and 9.f Bode diagrams) for the two extreme values of $\mathrm{M}$, one observes that the controller gain variations and the plant gain variations compensate for each other. This means an open loop cross-over frequency insensitive to the mass variations. Thus, the insensitivity of $\omega_{u}$ ensures the robustness of the rapidity (intrinsic property of hydropneumatic technology) and the constancy of the phase margin $M_{\Phi}$ ensures the robustness of the stability degree (intrinsic property of CRONE approach) towards the sprung mass variations. 


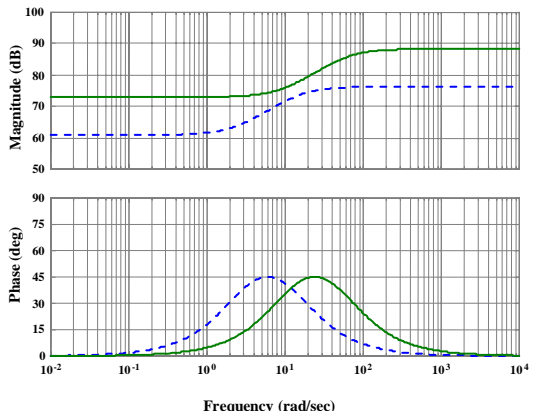

(a)
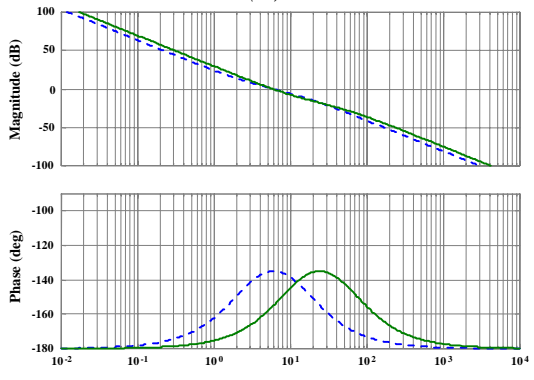

(c)

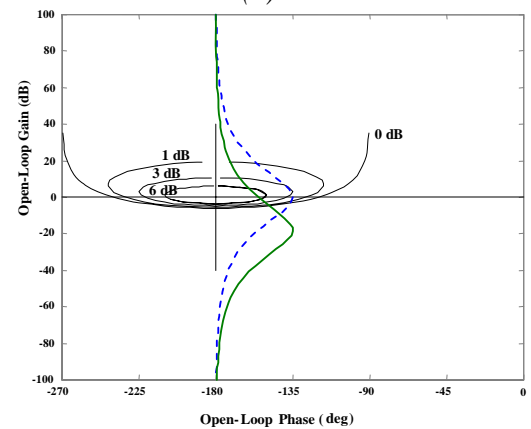

(e)

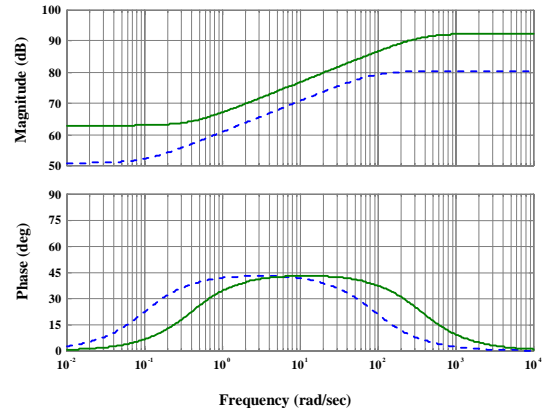

(b)

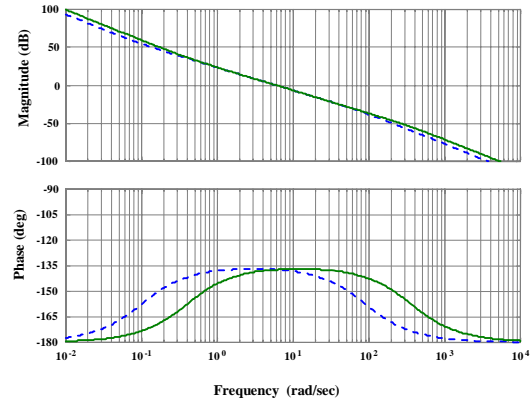

(d)

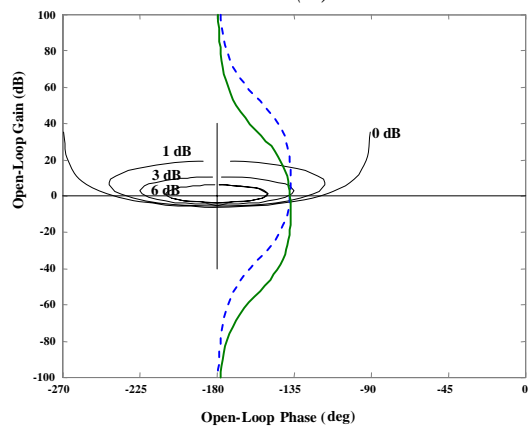

(f)

Fig. 9. Frequency responses obtained with the traditional suspension ((a), (c) and (e)) and the CRONE suspension ((b), (d) and (f)) for the extreme mass values $M$

These properties are illustrated figure 10. Figure 10 presents the time responses of a release test obtained with the traditional suspension (a) and with the CRONE suspension (b) for the two sprung mass extreme values (blue dotted line: $M=75 \mathrm{~kg}$, in green $M=150 \mathrm{~kg}$ ). The mass is initially hold in a high position and release at $t=0\left(z_{1}\left(0^{-}\right)=1\right)$. 


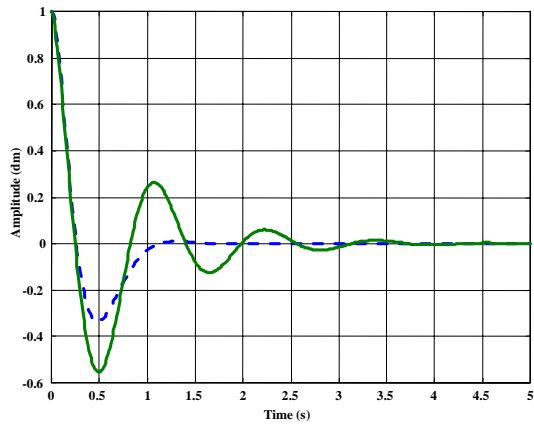

(a)

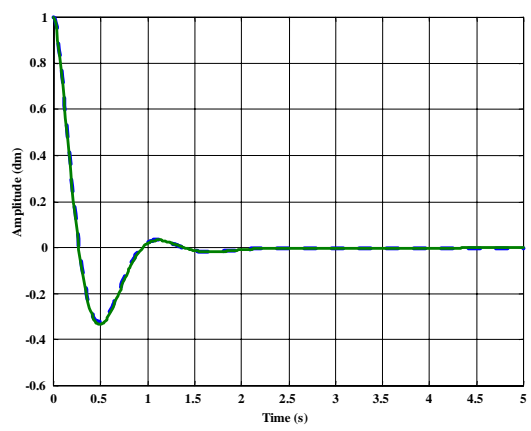

(b)

Fig. 10. Time responses of the traditional suspension (a) and of the CRONE suspension (b) with the two extreme sprung mass values $M$ (blue dotted line: $\mathrm{M}=75 \mathrm{~kg}$, in green $\mathrm{M}=150 \mathrm{~kg}$ )

\section{Conclusion}

The performances of the test bench presented in this article make it possible to highlight the interest of fractional derivative in vibration isolation. The performances obtained are remarkable, in particular when suspension CRONE is achieved in hydropneumatic technology from a method of synthesis based on the frequential recursivity. Indeed, the association of CRONE approach for the synthesis and of hydropneumatic technology for the achievement makes it possible to obtain the robustness of the stability degree, but also the robustness of the rapidity towards the mass variations. Two structures have been presented with the advantages and performance. An interesting point is that the gamma structure induces less technological parameters dispersion than the parallel arrangement of RC cells. This can allow to achieve more easily industrial applications of the CRONE suspension because of the standardization of the hydraulic accumulator in the gamma arrangement case. The next step of this work consists in taking into account tne component nonlinearities. The final objective is to find a design rule for vehicle hydraulic resistors which are nonlinear for functional reasons.

The principal industrial applications of this work are the automobile suspensions in particular with the Hydractive CRONE suspension [9]. It should be noted that the Hydractive CRONE suspension which presents three operating modes (a comfort mode, an intermediate mode and a safety mode) is the origin of the definition of a new class of systems, namely the Hybrid Fractional Dynamic Systems (HFDS) [10]. 


\section{References}

1. A. Le Mehauté (1991), Fractal Geometries, CRC Press INC Boca Raton - Ann Arbor - London.

2. B. Onaral and H.P. Schwan (1982), Linear and non linear properties of platinum electrode polarization, Part I, Frequency dependence at very low frequencies, Med. Bio . Eng. Comput., vol. 20, pp. 299-306.

3. X. Moreau, A. Oustaloup and M. Nouillant (1999), From analysis to synthesis of vehicle suspensions: the CRONE approach, Proceedings of ECC'99, Karlsruhe.

4. X. Moreau, C. Ramus-Serment and A. Oustaloup (2002), Fractional Differentiation in Passive Vibration Control; Special Issue on Fractional Calculus in the Journal of Nonlinear Dynamics, Vol. 29, pp. 343-362.

5. A. Oustaloup (1991), La commande CRONE ; Edition Hermès, Paris.

6. A. Oustaloup (1995), La dérivation non entière: théorie, synthèse et applications ; Edition Hermès, Paris.

7. Binder, R.C. (1973) Fluid Mechanics, Prentice-Hall, Inc. Englewood Cliffs, NJ.

8. Serrier, P., X. Moreau and A. Oustaloup (2005). Synthesis of a limited bandwidth fractional differentiator made in hydropneumatic technology. ASME International Design Engineering Technical Conferences \& Computers and Information in Engineering Conference, Long Beach, California, USA.

9. P. Serrier (2004), Synthèse fondée sur la récursivité fréquentielle d'un dérivateur d'ordre non entier borné en fréquence réalisé en technologie hydropneumatique - Application à la suspension CRONE Hydractive, Mémoire de stage MASTER EEA Recherche, Ecole Doctorale des Sciences Physiques et de l'Ingénieur de l'Université Bordeaux 1

10. O. Altet, C. Nouillant, X. Moreau and A. Oustaloup (2003), Hydractive CRONE suspension as hybrid system; International Journal of Hybrid System, Vol. 3, n² and 3, pp. 165-188. 